\title{
Heyting Valued Considerations on Some Fundamental Existence Theorems in Modern Analysis
}

By

\author{
Hirokazu NISHIMURA*
}

\begin{abstract}
Following the honorable tradition of Rousseau's [6] treatment of Weierstrass theorems in several complex variables, we give some other specimens of transfer from intuitionistic theorems to classical ones. The classical theorems which we deal with in this paper include the implicit function theorem and Cauchy's local existence theorem in ordinary differential equations.
\end{abstract}

\section{§1. Preamble}

Rousseau [6] has shown that an intuitionistic division theorem in one variable, interpreted in the Heyting valued set theory $V^{(\Omega)}$ with $\Omega$ being the topology $\mathscr{D}\left(\boldsymbol{C}^{n-1}\right)$ of $\boldsymbol{C}^{n-1}$, gives rise to the classical Weierstrass division theorem in $n$ variables. Following these lines, we will show that the inverse function theorem, demonstrated intuitionistically and interpreted in the Heyting valued set theory $V^{(\Omega)}$ with $\Omega$ being the topology of some appropriately chosen space of parameters, is nearly the classical implicit function theorem. Similarly it will be demonstrated that Cauchy's celebrated local existence theorem for ordinary differential equations, established intuitionistically and interpreted in the Heyting valued set theory $V^{(\Omega)}$ with $\Omega$ being the topology of some appropriately chosen space of parameters, gives rise to the classical theorems concerning the smooth dependence of local solutions on parameters and on initial conditions.

The organization of the paper goes as follows. After reviewing

Communicated by S. Takasu, December 2, 1987.

* Institute of Mathematics, University of Tsukuba, Ibaraki 305, Japan. 
some rudiments of Heyting valued set theory and elementary intuitionistic analysis in Section 2, we deal with the internal and external aspects of a simple fixed point theorem in Section 3. Section 4 is devoted to the internal and external aspects of the intuitionistic inverse function theorem, while Section 5 treats Cauchy's local existence theorem for ordinary differential equations both internally and externally. Most theorems and definitions are headed by (internal), (external), $(\mathrm{ZFC})$ or $\left(\mathrm{ZF}_{\mathrm{I}}\right)$ to stress their nature。 For technical reasons we deal almost exclusively with the complex or holomorphic case.

\section{§2. Preliminaries}

\subsection{Intuitionistic Set Theory}

By $\mathrm{ZF}_{\mathrm{I}}$ we mean a first-order intuitionistic theory with a unary relation symbol $\mathrm{E}$ and two binary relation symbols $\in$ and $=$ satisfying the following nonlogical axioms:

(A1) Equality axioms : $u=u$,

$$
\begin{aligned}
& u=v \rightarrow v=u, \\
& u=v \wedge \varphi(u) \rightarrow \varphi(v), \text { and }(\mathrm{E} u \bigvee \mathrm{E} v \rightarrow u=v) \rightarrow u=v 。
\end{aligned}
$$

(A2) Extensionality: $\dot{\forall} z(z \in u \leftrightarrow z \in v) \wedge(\mathrm{E} u \leftrightarrow \mathrm{E} v) \rightarrow u=v$ 。

(A3) Pairing: $\dot{\exists} z \dot{\forall} x(x \in z \leftrightarrow x=u \bigvee x=v)$ 。

(A4) Union: $\dot{\exists} v \dot{\forall} x(x \in v \leftrightarrow \dot{\exists} y \in u(x \in y))$.

(A5) Power sets : $\dot{\exists} v \dot{\forall} x(x \in v \leftrightarrow \dot{\forall} y \in x(y \in u))$.

(A6) $\in$-induction: $\dot{\forall} x(\dot{\forall} y \in x \varphi(y) \rightarrow \varphi(x)) \rightarrow \dot{\forall} x \varphi(x)$.

(A7) Infinity: $\dot{\exists} v(\dot{\exists} x \in v \wedge \dot{\forall} x \in v \dot{\ominus} y \in v(x \in y))$.

(A8) Separation: $\dot{\exists} v \dot{\forall} x(x \in v \leftrightarrow x \in u \wedge \varphi(x))$.

(A9) Collection: $\dot{\exists} v(\dot{\forall} x \in u \dot{\Xi} y \varphi(x, y) \rightarrow \dot{\forall} x \in u \dot{\exists} y \in v \varphi(x, y))$.

In the above list $\dot{\forall} x \ldots$ and $\dot{\exists} x \ldots$ are abbreviations of $\forall x$ $(\mathrm{E} x \rightarrow \cdots)$ and $\exists x(\mathrm{E} x \wedge \cdots)$. Since $\forall x$ and $\exists x$ will usually appear in these forms, we will often write $\forall x$ and $\exists x$ simply for $\dot{\forall} x$ and $\dot{\ominus} x$ 。

\subsection{Heyting Valued Models}

Let $V$ be a standard universe of ZFG and let $\Omega$ be a complete Heyting algebra. For each ordinal $\alpha$ we define $V_{\alpha}^{(\varrho)}$ inductively to 
be the set of all ordered pairs $\langle u, \mathrm{E} u\rangle$ such that:

(1) $\mathrm{E} u \in \Omega$;

(2) $\quad u$ is an $\Omega$-valued function defined on a subset $\mathscr{D}(u)$ of $V_{\beta}^{(\Omega)}$ for some ordinal $\beta<\alpha$ :

(3) $\forall x \in \mathscr{D}(u)(u(x) \leqq \mathrm{E} u \wedge \mathrm{E} x)$.

Now $V^{(\Omega)}$ is defined to be the class $\cup_{\alpha \in \text { on }} V_{\alpha}^{(\Omega)}$, which is to be called an $(\Omega$-valued) sheaf model, can be considered to be a Heyting valued model of $\mathrm{ZF}_{\mathrm{I}}$ by defining [Eu] with

(1) $\llbracket \mathrm{E} u \rrbracket=\mathrm{E} u$,

and by defining $\llbracket u \in v \rrbracket$ and $\llbracket u=v \rrbracket$ with the following simultaneous induction

(2) $\quad \llbracket u \in v \rrbracket=\bigvee_{y \in \mathscr{D}(v)}(v(y) \wedge \llbracket u=y \rrbracket)$,

(3) $\llbracket u=v \rrbracket=\wedge_{x \in \mathscr{D}(u)}\left(u(x) \rightarrow \llbracket x \in v \rrbracket \wedge \wedge_{y \in \mathscr{D}(v)}(v(y) \rightarrow \llbracket y \in u \rrbracket) \wedge(\mathrm{E} u \leftrightarrow\right.$ $\mathrm{E} v)$,

and then by assigning a Heyting value $\llbracket \varphi \rrbracket$ to each nonatomic sentence $\varphi$ inductively as follows :

(4) $\quad\left[\varphi_{1} \wedge \varphi_{2}\right]=\llbracket \varphi_{1} \rrbracket \wedge \llbracket \varphi_{2} \rrbracket$,

(5) $\llbracket \varphi_{1} \vee \varphi_{2} \rrbracket=\llbracket \varphi_{1} \rrbracket \vee \llbracket \varphi_{2} \rrbracket$,

(6) $\llbracket \varphi_{1} \rightarrow \varphi_{2} \rrbracket=\llbracket \varphi_{1} \rrbracket \rightarrow \llbracket \varphi_{2} \rrbracket$,

(7) $\llbracket \neg \varphi \rrbracket=\neg \llbracket \varphi \rrbracket$,

(8) $\quad \llbracket \exists x \varphi(x) \rrbracket=\bigvee_{x \in V}^{(\Omega)} \llbracket \varphi(x) \rrbracket$,

(9) $\quad\left[\forall x \varphi(x) \rrbracket=\bigwedge_{x \in V}^{(\Omega)} \llbracket \varphi(x) \rrbracket\right.$.

Now we have

Theorem 2.2.1. $V^{(\Omega)}$ is a model of $\mathrm{ZF}_{\mathrm{I}}$.

The class $V$ can be embedded into $V^{(\Omega)}$ by transfinite induction as follows :

$$
\check{y}=\{\langle\check{x}, 1\rangle \mid x \in y\} \text { and } \mathrm{E} \check{y}=1 \quad \text { for } y \in V \text {. }
$$

For $u \in V^{(\Omega)}$ and $p \in \Omega$, we define $u\left\lceil p\right.$ to be the element of $V^{(\Omega)}$ such that

$$
\begin{aligned}
& \mathscr{D}(u\lceil p)=\{x \Gamma p \mid x \in \mathscr{D}(u)\}, \\
& (u \Gamma p)(x \Gamma p)=\vee\{u(t) \wedge p \mid t \in \mathscr{D}(u), t\ulcorner p=x \Gamma p\} \text { for } x \in \mathscr{D}(u), \\
& \text { and } \mathrm{E}(u \Gamma p)=\mathrm{E} u \wedge p .
\end{aligned}
$$


In the sequel we implicitly identify $x, y \in V^{(\Omega)}$ time and again provided $\llbracket x=y \rrbracket=1$.

\subsection{Sheaves over Complete Heyting Algebras}

A presheaf over a complete Heyting algebra $Q$ is a triple $\langle S, \mathrm{E}, \Gamma\rangle$ of a set $S$ and two functions $\mathrm{E}: S \rightarrow \Omega$ and $\Gamma: S \times \Omega \rightarrow S$ with the following properties:

(1) $\quad a\ulcorner 0=\mathrm{b}\ulcorner 0$,

(2) $a\ulcorner\mathrm{E} a=a$,

(3) $\mathrm{E}(a\ulcorner p)=\mathrm{E} a \wedge p$,

(4) $\quad(a\ulcorner p)\ulcorner q=a \Gamma(p \wedge q)$.

For convenience we often say simply that $S$ is a presheaf over $\Omega$ without mentioning $\mathrm{E}$ and $\Gamma$ explicitly. Members $a, b$ of a presheaf $S$ over $\varrho$ are said to be compatible whenever $a\ulcorner\mathrm{E} b=b \Gamma \mathrm{E} a$. A subset $F$ of $S$ whose members are pairwise compatible is called compatible. A presheaf $S$ over $\Omega$ is called a sheaf over $\Omega$ if for any compatible subset $F$ of $S$ there exists a unique $g \in S$ such that:

(1) $f \in F$ implies $g\ulcorner\mathrm{E} f=f$,

(2) $\mathrm{E} g=\bigvee\{\mathrm{E} f \mid f \in F\}$.

The subset $\{a \in S \mid \mathrm{E} a=p\}$ is denoted by $\Gamma(p, S)$.

Theorem 2.3.1. For any $u \in V^{(\Omega)}, \tilde{u}=\left\{x \in V^{(\Omega)}, \llbracket x \in u \rrbracket=\mathrm{E} x\right\}$ is a sheaf to be called the sheaf represented by $u$. Conversely, for any sheaf $S$ over $\Omega$, there is an element $u \in V^{, \Omega}$ such that the sheaf $\tilde{u}$ represented by $u$ is isomorphic to $S$.

Similarly we have

Theorem 2.3.2. Let $u_{1}, u_{2} \in V^{(\Omega)}$. Then any function $f: u_{1} \rightarrow u_{2}$ in $V^{(\Omega)}$ renders a unique function $\tilde{f}: \tilde{u}_{1} \rightarrow \tilde{u}_{2}$ such that for each $a \in \tilde{u}_{1}$,

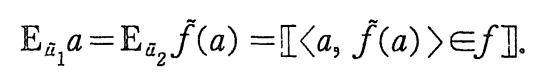

This gives a bijective correspondence between functions $f: u_{1} \rightarrow u_{2}$ in $V^{(\Omega)}$ and functions $g: \tilde{u}_{1} \rightarrow \tilde{u}_{2}$ such that

$$
\begin{aligned}
& \mathrm{E}_{\tilde{u}_{1}} a=\mathrm{E}_{\tilde{u}_{2}} g(a) \text { and } g(a) \Gamma p=g(a \Gamma p) \\
& \text { for any } p \in \Omega \text { and } a \in \tilde{u}_{1} \text {. }
\end{aligned}
$$




\subsection{Intuitionistic Linear Algebra}

Definition $\left(\mathrm{ZF}_{\mathrm{I}}\right)$. A relation $\neq$ on a set $S$ is called apartness if for any $a, b, c \in S$, we have:

(2) $\quad a=b \leftrightarrow \neg(a \neq b)$;

(3) $a \neq b \rightarrow a \neq c \bigvee b \neq c$.

Definition $\left(\mathrm{ZF}_{\mathrm{I}}\right)$. A ring $S$ with apartness $\neq$ is called an apartness ring if for $a, b, c \in S$, we have:

(1) $a \neq b \rightarrow a+c \neq b+c$;

(2) $a \neq b \wedge c \neq 0 \rightarrow a c \neq b c$.

Definition $\left(\mathrm{ZF}_{\mathrm{I}}\right)$. An apartness ring $S$ with 1 is called an apartness field if for any $a \in S$, we have:

(1) $\quad 1 \neq 0$;

(2) $\quad a \neq 0 \rightarrow \exists a^{-1}\left(a^{-1} \neq 0 \wedge a a^{-1}=1\right)$.

Definition $\left(\mathrm{ZF}_{1}\right)$. Let $F$ be an apartness field. Then an $F-$ module $T$ with apartness $\neq$ is called an apartness vector space over $F$ provided for any $a, a^{\prime} \in F$ and any $u, u^{\prime}, v, v^{\prime} \in T$, we have:

(1) $\quad a u \neq a^{\prime} u^{\prime} \rightarrow a \neq a^{\prime} \bigvee u \neq u^{\prime}$;

(2) $u+v \neq u^{\prime}+v^{\prime} \rightarrow u \neq u^{\prime} \bigvee v \neq v^{\prime}$.

Definition $\left(\mathrm{ZF}_{\mathrm{I}}\right)$. Let $T$ be an apartness vector space over an apartness field $F$. A finite set $\left\{e_{1}, \ldots, e_{n}\right\}$ of vectors is called an apartness basis if

(1) $\left\{e_{1}, \ldots, e_{n}\right\}$ is apartness linearly independent, i.e., $\forall\left\{a_{1}, \ldots, a_{n}\right\} \subset F\left(a_{i} \neq 0 \rightarrow a_{1} e_{1}+\cdots+a_{n} e_{n} \neq 0\right) ;$

(2) $T$ is generated by $\left\{e_{1}, \ldots, e_{n}\right\}, i_{\circ}$ e., $\forall u \in T \exists\left\{a_{1}, \ldots, a_{n}\right\} \subset F\left(u=a_{1} e_{1}+\cdots+a_{n} e_{n}\right)$.

Theorem 2.4.1 $\left(\mathrm{ZF}_{1}\right)$. Let $T$ be an apartness vector space over an apartness field $F_{0}$. If $\left\{e_{1}, \ldots, e_{m}\right\}$ and $\left\{e_{1}^{\prime}, \ldots, e_{n}^{\prime}\right\}$ are bases for $T$, then $m=n$ 。 
Definition $\left(\mathrm{ZF}_{\mathrm{I}}\right)$. Let $T_{1}$ and $T_{2}$ be apartness vector spaces over an apartness field $F$. A mapping $\varphi: T_{1} \rightarrow T_{2}$ is called a homomorphism if

$$
\forall u, v \in T_{1} \forall a, b \in F(\varphi(a u+b v)=a \varphi(u)+b \varphi(v)) .
$$

A homomorphism $\varphi: T_{1} \rightarrow T_{2}$ is called an embedding if

$$
\forall u, v \in T_{1}(\varphi(u)=\varphi(v) \rightarrow u=v) \text {. }
$$

A homomorphism $\varphi: T_{1} \rightarrow T_{2}$ is called an apartness embedding if

$$
\forall u, v \in T_{1}(u \neq v \rightarrow \varphi(u) \neq \varphi(v)) .
$$

An (apartness) embedding $\varphi: T_{1} \rightarrow T_{2}$ is called an (apartness) isomorphism if

$$
\forall v \in T_{2} \exists u \in T_{1}(\varphi(u)=v) \text { 。 }
$$

The determinant of an $n \times n$ matrix is defined to be the wellknown polynomial of $n^{2}$ variables. And we have

Theorem 2.4.2 $\left(\mathrm{ZF}_{\mathrm{I}}\right)$. If $F$ is an apartness field, $h: F^{n} \rightarrow F^{n}$ is a homomorphism and $M$ is the matrix of $h$, then $M$ is invertable iff $\operatorname{det} M \neq 0$, where $\operatorname{det} M$ is the determinant of $M$.

\subsection{Elementary Intuitionistic Analysis}

In this subsection we review some rudiments of intuitionistic analysis which are indispensable for our later discussion. Now that such a readable exposition as Bishop and Bridges [1] is available, our exposition here can hopefully be sketchy.

Let $X$ be a topological space with topology $\Omega=\mathfrak{D}(X)$. Then the usual Dedekind construction in $V^{(\Omega)}$ gives the system $\mathbb{R}^{(\Omega)}$ which is externally the sheaf of real-valued continuous functions on $X$. Similarly $\mathbb{C}^{(\Omega)}=\mathbb{R}^{(\Omega)}+i \mathbb{R}^{(\Omega)}$ is externally the sheaf of complex-valued continuous functions on $X$. There is a standard apartness relation $\neq$ in $\mathbb{R}^{(\Omega)} ; x \neq y$ iff $x<y$ or $x>y$. An apartnes subfield $\Re$ of $\mathbb{R}^{(\Omega)}$ containing is called a real-like field if $\mathfrak{R}$ is complete (i. e., every Cauchy sequence converges).

Elementary real analysis, including a slightly weaker version of the mean value theorem, can be developed internally by using any real-like field $\Re$ in place of $\mathbb{R}$. Notice that the completeness of $\Re$ 
plays a crucial role in defining the familiar function exp as the limit of an infinite series and in defining the Riemann integrals of continuous functions as the limit of finite sums. Similarly an apartness subfield $\mathbb{C}$ of $\mathbb{C}^{(\Omega)}$ containing $\check{C}$ is called a complex-like field if $\mathfrak{F}$ is complete. The most important complex-like field that we often encounter is $\boldsymbol{H}$ whose representing sheaf is the sheaf of holomorphic functions on $X$, where $X$ is surely required to be a complex manifold or the like in such a case. For all the variety of real-like and complex-like fields, we have the following.

Theorem 2.5.1 (ZFC). If $\Omega$ is the topology $\mathcal{D}(X)$ of a topological space $X$, then for any real-like field $\Re$ in $\boldsymbol{V}^{(\Omega)}$, the totality of open subsets of $\Re^{n}$ is externally the totality of open subsets of $X \times \boldsymbol{R}^{n}$. Similarly, for any complex-like field ${ }^{5}$ in $\boldsymbol{V}^{(\Omega)}$, the totality of open subsets of $\mathbb{E}^{n}$ is externally the totality of open subsets of $X \times C^{n}$.

Proof. See Rousseau [6] or Takeuti [8].

Similarly we have

Theorem 2.5.2 (ZFG). If $\Omega$ is the topology $\mathscr{Q}(X)$ of a topological space $X$, then, for any real-like field $\Re$, the sheaf of $\Re$-valued continuous functions on $\Re^{n}$ is externally the sheaf of real-valued continuous functions on $X \times \boldsymbol{R}^{n}$. Similarly, for any complex-like field $\mathfrak{E}$, the sheaf of $\mathfrak{\mathbb { E } \text { -valued }}$ continuous functions on $\mathbb{E}^{n}$ is externally the sheaf of complex-valued continuous functions on $X \times C^{n}$.

Proof. See Rousseau [6] or Takeuti [8].

Theorem 2.5.3 (ZFC). If $\Omega$ is the topology $\subseteq(U)$ of an open subset $U$ of $\mathbb{C}^{m}$, then the sheaf of holomorphic functions on $\boldsymbol{H}^{n}$ is externally the sheaf of holomorphic functions on $\boldsymbol{C}^{m+n}$.

Proof. See Rousseau [6] or Takeuti [8].

The following definition is necessary in Sections 4 and 5 . 
Definition (internal). Let $\Omega$ be the topology $\mathscr{Q}(U)$ of an open subset $U$ of $\mathbb{C}^{n}$. Then we need the following definition in $V^{(\Omega)}$ : For any linear mapping $T: \mathbb{H}^{p} \rightarrow \mathbb{H}^{q}$ and any positive number $M$, we write $\|T\| \leq M$ if $\|T x\| \leq M\|x\|$ for any $x \in \mathbb{H}^{p}$, where $\|x\|$ is the square root of the sum of the squares of the components of $x$ as usual.

The following style of the internal mean value theorem will be required in Sections 4 and 5.

Theorem 2.5.4 (internal). Let $\Omega$ be the topology $D(U)$ of an open subset $U$ of $\mathbb{C}^{n}$. Then we have following in $V^{(\Omega)}$ : For any holomorphic mapping $f$ from an open ball $W$ of $\mathbb{H}^{p}$ to $\mathbb{H}^{q}$ and any $x, y \in W$, if $\| D f$ $(\xi x+(1-\xi) y) \| \leq M(0 \leq \xi \leq 1)$ for some positive number $M$, then $\| f(x)$ $-f(y)\|\leq M\| x-y \|$, where $D f(z)$ for $z \in W$ is the derivative of $f$ at $z$ and so is a linear mapping from $\mathbb{H}^{p}$ to $\mathbb{H}^{q}$ whose matrix is the so-called Jacobian matrix of $f$ at $z$.

Proof. This follows readily from the internal version of the mean value theorem, for which the reader is referred to Bishop and Bridges $[1 ; \mathrm{p} .48]$.

Q.E.D.

The following theorem is indispensable in Section 4.

Theorem 2.5.5 (ZFC). Let $\Omega$ be the topology $\mathcal{O}(U)$ of an open subset $U$ of $\mathbb{C}^{n}$. Let $f$ be a holomorphic function from an open subset $W$ of $\mathbb{C}^{n+p}=\mathbb{C}^{n} \times \mathbb{C}^{p}$ to $\mathbb{C}^{q}$ and let $\hat{f}$ be its internal correspondent in $V^{(\Omega)}$ under Theorem 2.5.3. Then the internal function $D \hat{f}$ is externally the function $D_{2} f$, where $D_{2} f$ is the partial derivative with respect to the second variable and so determines a linear mapping from $\mathbb{C}^{b}$ to $\mathbb{C}^{q}$ at each point of $W$.

The following definition is indispensable in the proof of Theorem 5.4.

Definition (internal). Let $\Omega$ be the topology $\subseteq(U)$ of an open subset $U$ of $\mathbb{C}^{n}$. Then we need the following definitions in $V^{(\Omega)}$ : 
Let $f, g$ be bounded functions from the same subset $W$ of $\boldsymbol{H}^{p}$ to $\boldsymbol{H}^{q}$. We write $\|f\| \leq\|g\|$ if, for any positive number $\eta,\|g(x)\| \leq \eta$ for any $x \in W$ implies $\|f(x)\| \leq \eta$ for any $x \in W$. Similarly we write $\alpha \leq \beta\|g\|$, where $\alpha$ and $\beta$ are positive numbers, if, for any positive number $\eta,\|g(x)\| \leq \eta$ for any $x \in W$ implies $\alpha \leq \beta \eta$.

The following definition and theorem will be used in the proof of Theorem 5.3 .

Definition (internal). Let $U$ be an open subset of $C^{n}$ with its relative topology $\Omega=\mathscr{O}(U)$. Then we need the following definition in $V^{(\Omega)}$ : Let $W$ be an open subset of $\mathbb{H}^{p}$ and $A \subset W$ be an open ball. Then $A$ is said to be well contained in $W$ (notation: $A \in W$ ) if there is an open ball $A^{\prime} \subset W$ with the same center and a larger radius.

Theorem 2.5.6 (internal). Let $U$ be an open subset of $\mathbb{C}^{n}$ with its relative topology $\subseteq(U)$. Then we have the following in $V^{(\Omega)}:$ Let $f$ be a continuous function from an open subset $J$ of $\boldsymbol{H}^{p}$ to an open ball $W$ of $H^{q}$ with center $z_{0}$. Then, for any open ball $J^{\prime} \in J$, there is an open ball $W^{\prime} \in W$ with center $z_{0}$ such that $f\left(J^{\prime}\right) \subset W^{\prime}$.

So far we have succeeded in proving this theorem only externally, so we would be glad to see someone try to establish Theorem 5. 3 without resort to Theorem 2.5.6.

\section{\$3. Fixed Point Theorem}

The main purpose of the present section is to establish the following well-known classical theorem as the external reformulation of an intuitionistic simpler version. The method is the prototype of all the succeeding sections.

Theorem 3.1 (external)。 Let $X$ be a topological space, $E$ be a (real or complex) Banach space, and $U$ be an open ball with center 0 and radius $\alpha$. Let $v$ be a continuous mapping from $X \times U$ to $E$ such that

$$
\left\|v\left(x, y_{1}\right)-v\left(x, y_{2}\right)\right\| \leq k\left\|y_{1}-y_{2}\right\|
$$


for any $x \in X, y_{1}, y_{2} \in U$, where $k$ is a constant such that $0 \leq k<1$. Then, if $\|v(x, 0)\|<\alpha(1-k)$ for any $x \in X$, there exists a unique continuous mapping $f: X \rightarrow U$ such that

$$
f(x)=v(x, f(x))
$$

for any $x \in X$.

We can derive the above theorem from the following internal one, whose external counterpart is usually regarded as an easy corollary of the above one.

Theorem 3.2 (internal). Let $X$ be a topological space with topology $\Omega=\mathfrak{O}(X)$. Then we have the following in $V^{(\Omega)}:$ Let $E$ be a Banach space over $\mathbb{R}^{(\Omega)}$ or $\mathbb{C}^{(\Omega)}$ and $U$ be an open ball with center 0 and radius $\alpha_{\text {. }}$ Let $v$ be a mapping from $U$ to $E$ such that

$$
\left\|v\left(y_{1}\right)-v\left(y_{2}\right)\right\| \leq k\left\|y_{1}-y_{2}\right\|
$$

for any $y_{1}, y_{2} \in U$, where $k$ is a constant such that $0 \leq k<1$. Then, if $\|v(0)\|<\alpha(1-k)$, there is a unique element $z \in U$ such that $z=v(z)$.

Proof. The standard proof of successive approximation still holds literatim in $V^{(\Omega)}$. The proof is divided into uniqueness and existence parts.

(a) uniqueness: Let $w, z \in U$ be such that $v(z)=z$ and $v(w)=w$ 。 Then we have

$$
\|z-w\|=\|v(z)-v(w)\| \leq k\|z-w\| .
$$

Since $k<1$ by assumption, it must be the case that $z=w$.

(b) existence: We would like to define inductively a sequence $\left(y_{n}\right)$ in $U$ such that $y_{0}=0$ and $y_{n}=v\left(y_{n-1}\right)$ for any $n \geq 1$. Suppose that a finite sequence $\left(y_{p}\right)_{0 \leq p \leq n}$ in $U$ is defined with $y_{0}=0$ and $y_{p}=v\left(y_{p-1}\right)$ $(1 \leq p \leq n)$, then we have by induction that

$$
\text { (*) }\left\|y_{p}-y_{p-1}\right\| \leq k^{p-1}\left\|y_{1}\right\| \text {. }
$$

Therefore

$$
\text { (**) } \quad\left\|y_{p}\right\| \leq\left(1+k+\cdots+k^{p-1}\right)\left\|y_{1}\right\| \leq\left\|y_{1}\right\| /(1-k)<\alpha_{0}
$$

In particular, $\left\|y_{n}\right\|<\alpha$, and so we can safely define $y_{n+1}$ to be $v\left(y_{n}\right)$. It is also easy, by using $(*)$ and $(* *)$, to see that the sequence $\left(y_{n}\right)$ defined inductively in the above manner is a Cauchy sequence and its limit $z=\lim _{n \rightarrow \infty} y_{n}$ is the desired fixed point. $\quad$ Q. E. D. 
Now we would like to transfer this internal fixed point theorem without parameters to the external fixed point theorem with parameters.

Proof of Theorem 3.1. Let $\Omega=\mathscr{D}(X)$ and $E^{(\Omega)}$ be an internal Banach space in $V^{(\Omega)}$ represented by the sheaf of continuous functions from $X$ to $E$. We know well that there is a bijective correspondence between external open sets of $X \times E$ and internal open sets of $E^{(\Omega)}$. In particular, $X \times U$ corresponds to the internal open ball $U^{(\Omega)}$ with center 0 and radius $\check{\alpha}$. We know well also that there is a bijective correspondence between external continuous functions from $X \times U$ to $E$ and internal continuous functions from $U^{(\Omega)}$ to $E^{(\Omega)}$. In particular, the external function $v$ corresponds to the internal function $V^{(\Omega)}: U^{(\Omega)} \rightarrow E^{(\Omega)}$. It is easy to see that these internalized entities satisfy the conditions of Theorem 3.2. Indeed we have in $V^{(\Omega)}$

(I) $\left\|v^{(\rho)}\left(y_{1}\right)-v^{(\Omega)}\left(y_{2}\right)\right\| \leq \check{k}\left\|y_{1}-y_{2}\right\|$ for any $y_{1}, y_{2} \in U^{(\rho)}$;

(II) $\left\|v^{(\Omega)}(0)\right\|<\check{\alpha}(1-\check{k})$.

Thus the conclusion of Theorem 3.1 follows from the externalization of the conclusion of Theorem 3.2. $\quad$ Q.E.D.

We conclude this section with three comments.

(I) Not only the proof of Theorem 3.2 but also the derivation of Theorem 3.1 can be carried out even internally.

(II) Theorem 3.2 still holds even if we replace $\boldsymbol{R}^{(\Omega)}$ or $\boldsymbol{C}^{(\Omega)}$ by any real-like or complex-like field.

(III) Theorem 3.2 still holds with obvious modifications for complete quasinormed linear spaces. For the definition of a quasinormed linear space and its related concepts, the reader is referred to Bishop and Bridges [1; Chap. 7, §5].

\section{\$4. Implicit Function Theorem}

Let us begin this section with an internal version of the wellknown inverse function theorem. 
Theorem 4.1 (internal). Let $U$ be an open subset of $\mathbb{C}^{q}$ with its relative topology $\Omega=\mathfrak{O}(U)$. Then we have the following in $V^{(\Omega)}:$ Let $f$ be a holomorphic mapping from an open subset $W \subset \mathbb{H}^{p}$ to $\mathbb{H}^{p}$. Let $y_{0} \in W$ such that $D f\left(y_{0}\right)$ is invertible. Then there is an open neighborhood $W_{0}$ of $y_{0}$ and an open neighborhood $W_{1}$ of $z_{0}=f\left(y_{0}\right)$ such that $f$ is bijective from $W_{0}$ to $W_{1}$ with a holomorphic inverse.

Proof. We can choose positive numbers $M, \alpha, \beta$ satisfying the following conditions :

( I ) $\left\|D f\left(y_{0}\right)\right\| \leq M$;

(II) The open ball of $\mathbb{H}^{p}$ with center $y_{0}$ and radius $\alpha$ is contained in $W$;

(III) $\left\|D f(y)-D f\left(y_{0}\right)\right\| \leq \frac{1}{2 M}$ for any $y \in H^{p}$ such that $\left\|y-y_{0}\right\|$ $<\alpha$

(IV) $\left\|D f\left(y_{0}\right)^{-1}\left(f\left(y_{0}\right)-z\right)\right\|<\frac{\alpha}{2}$ for any $z \in H^{p}$ such that $\left\|z-z_{0}\right\|$ $<\beta$.

Let $v$ be a function to be defined as follows:

$$
v(z, y)=y-D f\left(y_{0}\right)^{-1}(f(y)-z)
$$

for any $y, z \in \mathbb{H}^{p}$ such that $\left\|y-y_{0}\right\|<\alpha$ and $\left\|z-z_{0}\right\|<\beta$. Then it is easy to see that $f(y)=z$ iff $v(z, y)=y$. Since

$$
\left\|v\left(z, y_{0}\right)\right\|=\left\|-D f\left(y_{0}\right)^{-1}\left(f\left(y_{0}\right)-z\right)\right\|<\frac{\alpha}{2}
$$

and

$$
\begin{aligned}
& \left\|v\left(z, y_{1}\right)-v\left(z, y_{2}\right)\right\| \\
= & \left\|y_{1}-D f\left(y_{0}\right)^{-1}\left(f\left(y_{1}\right)-z\right)-y_{2}+D f\left(y_{0}\right)^{-1}\left(f\left(y_{2}\right)-z\right)\right\| \\
= & \left\|D f\left(y_{0}\right)^{-1}\left(f\left(y_{2}\right)-f\left(y_{1}\right)\right)-D f\left(y_{0}\right)^{-1} D f\left(y_{0}\right)\left(y_{2}-y_{1}\right)\right\| \\
\leq & M\left\|f\left(y_{2}\right)-f\left(y_{1}\right)-D f\left(y_{0}\right)\left(y_{2}-y_{1}\right)\right\| \\
\leq & \frac{1}{2}\left\|y_{2}-y_{1}\right\|,
\end{aligned}
$$

we can apply an internal version of Theorem 3.1 and the desired result follows directly only by letting $W_{1}$ be the open ball with center $z_{0}$ and radius $\beta$ and $w_{0}$ be the inverse image $f^{-1}\left(W_{1}\right)$ of $W_{1}$ under $f$.

Q.E.D.

The external dividend of this theorem goes as follows. 
Theorem 4.2 (external). Let $f$ be a holomorphic mapping from an open subset $A \subset C^{p+q}=\mathbb{C}^{q} \times \mathbb{C}^{p}$ to $\mathbb{C}^{p}$. Let $\left(x_{0}, y_{0}\right) \in W$ be a point such that $D f\left(x_{0}, y_{0}\right)$ is invertible. Then, for any holomorphic function $v$ defined on an open neighborhood $U_{1}$ of $x_{0}$ with values in $\mathbb{C}^{p}$ such that $v\left(x_{0}\right)=f\left(x_{0}, y_{0}\right)$, there exist an open neighborhood $U_{0}$ of $x_{0}$ contained in $U_{1}$ and an open neighborhood $A_{0}$ of $\left(x_{0}, y_{0}\right)$ such that there is a unique holomorphic function $u: U_{0} \rightarrow \mathbb{C}^{p}$ satisfying $u\left(x_{0}\right)=y_{0},(x, u(x)) \in A_{0}$ and $f(x, u(x))=v(x)$ for any $x \in U_{0}$ 。

Proof. Take any open neighborhood $U$ of $x_{0}$ such that $D_{2} f\left(x, y_{0}\right)$ is invertible for any $x \in U$, and then apply Theorem 4.1 in $V^{(\Omega)}$ with $\Omega=\mathfrak{Q}(U)$.

Q.E. D.

\section{§5. Cauchy's Local Existence Theorem}

We begin this section with an internal version of Gronwall's lemma.

Theorem 5. 1 (internal). Let $X$ be a topological space with topology $\Omega=\$(X)$. Then we have the following in $V^{(\Omega)}$ : Let $\varphi(t), \psi(t)$ and $w(t)$ are continuous $\mathbb{R}^{(\Omega)}$-valued functions on a closed interval $[a, b]$ with $\phi(t)$ $\geq 0$. Then, if

$$
\text { (5.1.1) } \quad w(t) \leq \varphi(t)+\int_{a}^{t} \phi(s) w(s) d s \quad(a \leq t \leq b)
$$

then we have

$$
w(t) \leq \varphi(t)+\int_{a}^{t} \psi(s) \varphi(s) \exp \left(\int_{s}^{t} \psi(\sigma) d \sigma\right) d s \quad(a \leq t \leq b) .
$$

Proof. The standard proof holds literatim internally. Let $v(t)=$ $\int_{a}^{t} \phi(s) w(s) d s$. Since $v^{\prime}(t)=\phi(t) w(t)$ and $\phi(t) \geq 0, \quad(5.1 .1)$ yields

$$
v^{\prime}(t) \leq \phi(t) \varphi(t)+\phi(t) v(t)
$$

By multiplying $\exp \left(-\int_{a}^{t} \phi(\sigma) d \sigma\right)$ on both sides of (5.1.3) and transporting the second term of the right hand to the left, we have

$$
\frac{d}{d t}\left(\nu(t) \exp \left(-\int_{a}^{t} \psi(\sigma) d \sigma\right)\right) \leq \psi(t) \varphi(t) \exp \left(-\int_{a}^{t} \psi(\sigma) d \sigma\right) .
$$


Therefore, by integrating both sides of (5.1.4), we have

$$
v(t) \exp \left(-\int_{a}^{t} \psi(\sigma) d \sigma\right) \leq \int_{a}^{t} \psi(s) \varphi(s) \exp \left(-\int_{a}^{s} \psi(\sigma) d \sigma\right) d s,
$$

which yields

$$
v(t) \leq \int_{a}^{t} \psi(s) \varphi(s) \exp \left(\int_{s}^{t} \psi(\sigma) d \sigma\right) d s .
$$

Thus the desired inequality (5.1.2) follows.

Q. E. D.

Proposition 5.2 (internal). Let $U$ be an open subset of $\mathbb{C}^{q}$ with its relative topology $\Omega=\mathscr{Q}(U)$. Then we have the following in $V^{(\Omega)}$ : Let $f$ be a holomorphic function from $I \times W$ to $\mathbb{H}^{p}$, where $I$ is an open ball in $\mathbb{H}$ and $W$ is an open ball of $\mathbb{H}^{p}$. Then a holomorphic function $u: J \rightarrow W$, where $J \subset I$ is an open ball with center $t_{0}$, is a solution of the differential equation

$$
x^{\prime}=f(t, x)
$$

iff

(5.2.2) $u(t)=u\left(t_{0}\right)+\int_{t_{0}}^{t} f(s, u(s)) d s$,

where the integration is taken along the linear path $\xi \mapsto t_{0}+\xi\left(t-t_{0}\right)$ $(0 \leq \xi \leq 1)$.

Just as in the standard case, Gronwall's inequality gives the uniqueness theorem for ordinary differential equations.

Theorem 5. 3 (internal)。 Let $U$ be an open subset of $\mathbb{C}^{q}$ with its relative topology $\Omega=\mathfrak{D}(U)$. Then we have the following in $V^{(\Omega)}$ : Let $u$, $v$ be two solutions of $(5.2 .1)$ defined on the same open ball $J$ with center $t_{0}$. If $u\left(t_{0}\right)=v\left(t_{0}\right)$, then $u=v$ on $J$.

Proof. Let $J^{\prime} \Subset J$ be an arbitrary open ball containing $t_{0}$. Then there is an open ball $W^{\prime} \Subset W$ such that $u(t), v(t) \in W^{\prime}$ for any $t \in J^{\prime}$. Then there is a positive number $M$ such that $\left\|D_{2} f(t, x)\right\|$ $\leq M$ for any $t \in J^{\prime}, x \in W^{\prime}$. Since

$$
\begin{aligned}
& \|u(t)-v(t)\| \\
= & \left\|\int_{t 0}^{t}\{f(s, u(s))-f(s, v(s))\} d s\right\|
\end{aligned}
$$




$$
\begin{aligned}
\leq & \| \int_{0}^{\xi}\left\{f\left(t_{0}+\eta\left(t-t_{0}\right), u\left(t_{0}+\eta\left(t-t_{0}\right)\right)\right)-f\left(t_{0}+\eta\left(t-t_{0}\right), v\left(t_{0}+\eta\left(t-t_{0}\right)\right)\right)\right\} \\
& \left(t-t_{0}\right) d \eta \| \\
\leq & \int_{0}^{\xi} M\left\|t-t_{0}\right\|\left\|u\left(t_{0}+\eta\left(t-t_{0}\right)\right)-v\left(t_{0}+\eta\left(t-t_{0}\right)\right)\right\| d \eta,
\end{aligned}
$$

we can apply Theorem 5.1 to convince ourself that $u=v$ on $J^{\prime}$. Since $J^{\prime} \Subset J$ was an arbitrary open ball containing $t_{0}$, the proof is complete.

Q.E.D.

Now we would like to deal with an internal version of Cauchy's local existence theorem for ordinary differential equations.

Theorem 5.4 (internal). Let $U$ be an open subset of $\mathbb{C}^{q}$ with its relative topology $\Omega=\subseteq(U)$. Then we have the following in $V^{(\Omega)}$ : For any $t_{0} \in I$ and any $x_{0} \in W$, there exists an open ball $J \subset I$ wit' 2 center $t_{0}$ such that there is on $J$ a unique solution $u$ of (5.2.1) such that $u\left(t_{0}\right)=x_{0}$.

Proof. The uniqueness part was dealt with in Theorem 5.3, so we have only to deal with the existence part. We can take an open ball $J_{a} \subset I$ with center $t_{0}$ and radius a and an open ball $B \subset W$ with center $x_{0}$ and radius $b$ such that there are positive numbers $M, k$ satisfying $\|f(t, x)\| \leq M$ and $\left\|D_{2} f(t, x)\right\| \leq k$ for any $(t, x) \in J_{a} \times B$. Let $r$ be an arbitrary positive number such that $r<b /(M+k b)$ and $r<a$. Then $k r<1$ and $M \mathrm{r}<b(1-k r)$. Let $J_{r}$ be an open ball with center $t_{0}$ and radius $r$. Let $V_{r}$ be the space of all continuous functions $y: J_{r} \rightarrow \boldsymbol{H}^{p}$ such that $\left\|x_{0}-y\right\|<r$, where $x_{0}$ is regarded as the constant function $t \mapsto x_{0}$. Then, for any $y, y_{1}, y_{2} \in V_{r}$ and any $t \in J_{r}$, we have

$$
\begin{aligned}
& \left\|\int_{t_{0}}^{t} f\left(s, y_{1}(s)\right)-f\left(s, y_{2}(s)\right)\right\| \leq k r\left\|y_{1}-y_{2}\right\|, \text { and } \\
& \left\|\int_{t_{0}}^{t} f(s, y(s))\right\| \leq M r
\end{aligned}
$$

Thus we can apply the method of Theorem 3.2 to the function $g$ on $V_{r}$ such that $g(y)$ is the function $t \mapsto x_{0}+\int_{t_{0}}^{t} f(s, y(s)) d s$.

Q.E.D. 
The external dividend of the above internal theorem are the following two theorems concerning the dependence of a solution of $x^{\prime}=f(t, x)$ on parameters and on initial conditions respectively.

Theorem 5.5 (external). Let $I \subset \mathbb{C}$ be an open ball, $W \subset \mathbb{C}^{p}$ be an open ball and $P \subset C^{q}$ be an open ball. Let $f$ be a holomorphic mapping from $I \times W \times P$ to $\mathbb{C}^{p}$. Then, for any $\left(t_{0}, x_{0}, z_{0}\right) \in I \times W \times P$, there exist an open ball $J \subset I$ with center $t_{0}$ and an open ball $T \subset P$ with center $z_{0}$ such that there is a unique holomorphic function $u: J \times P \rightarrow \mathbb{C}^{p}$ satisfying $u\left(t_{0}, z\right)=x_{0}$ for any $z \in P$ and $u^{\prime}(t)=f(t, u(t), z)$ for any $(t, z) \in J \times P$ 。

Proof. Apply Theorem 5.4 in $V^{(\Omega)}$ with $\Omega=\mathfrak{D}(P)$ Q Q.E.D.

Theorem 5.6 (external). Let $f$ be a holomorphic function from $I \times W$ to $\mathbb{C}^{p}$, where $I$ is an open ball in $\mathbb{C}$ and $W$ is an open ball in $\mathbb{C}^{p}$. Then, for any $(a, b) \in I \times W$, there exist an open ball $J \subset I$ with center $a$ and an open ball $U \subset W$ with center $b$ such that there is a unique holomorphic function $u: J \times J \times U \rightarrow \mathbb{C}^{p}$ satisfying $u\left(t_{0}, t_{0}, x_{0}\right)=x_{0}$ for any $\left(t_{0}, x_{0}\right) \in J \times U$ and $u^{\prime}\left(t, t_{0}, x_{0}\right)=f\left(t, u^{\prime}\left(t, t_{0}, x_{0}\right)\right)$ for any $\left(t, t_{0}, x_{0}\right) \in$ $J \times J \times U$, where $u^{\prime}$ is the derivative of $u$ with respect to the first variable.

Proof. Apply Theorem 5.4 in $V^{(\Omega)}$ with $\Omega=\mathfrak{O}(I \times W)$, where $t_{0}$ in that theorem is represented externally by the function $(t, x) \mapsto t$ and $x_{0}$ in that theorem is represented externally by the function $(t, x) \mapsto x$.

Q.E.D.

\section{References}

[1] Bishop, E. and Bridges, D., Constructive analysis, Springer, Berlin and Heidelberg, 1985.

[2] Dieudonné, J., Foundations of modern analysis, Academic Press, New York and London, 1969.

[3] Grayson, R. J., Concepts of general topology in constructive mathematics and in sheaves, Annals of Mathematical Logic, 20 (1981), 1-41.

[4] Concepts of general topology in constructive mathematics and in sheaves, II, Annals of Mathematical Logic, 23 (1982), 55-98.

[5] Nishimura, H., Heyting valued set theory and fibre bundles, Publ. RIMS, 24 (1988), 225-247.

[6] Rousseau, C., Topos theory and complex analysis, Lecture Notes in Mathematics, 753 (1977), Springer, Berlin, 623-659. 
[7] Ruitenberg, W. B. G., Intuitionistic algebra, theory and sheaf models, dissertation, 1982.

[8] Takeuti, G., Intuitionistic set theory (in Japanese), Kinokuniya, Tokyo, 1980.

[9] Takeuti, G. and Titani, S., Heyting valued universes of intuitionistic set theory, Lecture Notes in Mathematics, 891 (1980), Springer, Berlin, 189-306.

[10] Globalization of intuitionistic set theory, Annals of Pure and Applied Logic, 33 (1987), 195-211.

[11] , Global intuitionistic analysis, preprint. 
